\title{
Brain Tau Imaging: Food and Drug Administration Approval of ${ }^{18}$ F-Flortaucipir Injection
}

\author{
Venkata S. Mattay ${ }^{1}$, Anthony F. Fotenos ${ }^{1}$, Charles J. Ganley ${ }^{2}$, and Libero Marzella ${ }^{1}$ \\ ${ }^{1}$ Division of Imaging and Radiation Medicine, Center for Drug Evaluation and Research, Food and Drug Administration, Silver \\ Spring, Maryland; and ${ }^{2}$ Office of Specialty Medicine, Center for Drug Evaluation and Research, Food and Drug Administration, \\ Silver Spring, Maryland
}

A lzheimer disease (AD) is the most common of neurodegenerative dementias. Currently, there are more than 5.8 million Americans with $\mathrm{AD}$. Worldwide, there are at least 50 million people with $\mathrm{AD}$ or other dementias, and these numbers are projected to triple by 2050 (1).

Timely and accurate diagnosis of AD can guide patients and their families through an anxious and uncertain experience by helping them anticipate future needs, including monitoring for progression, safety, and functional assessment. The accuracy of a clinical diagnosis of AD by dementia experts is modest (2), and a definitive diagnosis of $\mathrm{AD}$ can be established only by demonstrating the presence of $\beta$-amyloid plaques and tau-neurofibrillary tangles (NFTs) in the brain (3).

The Food and Drug Administration (FDA) previously approved radioactive PET tracers $\left({ }^{18} \mathrm{~F}\right.$-florbetapir, ${ }^{18} \mathrm{~F}$-florbetaben, and ${ }^{18} \mathrm{~F}$ flutemetamol) to assess the presence of $\beta$-amyloid plaques in the brain. However, by itself, the presence of $\beta$-amyloid in a brain PET scan is not sufficient for a diagnosis of AD. Confirmation of the presence of NFTs, the second pathologic hallmark of $\mathrm{AD}$, is necessary to definitively diagnose AD. The NFTs in AD typically exist as paired helical filaments and accumulate in a distinct spatiotemporal pattern (3). Accumulation starts in the transentorhinal cortex before spreading to the medial and inferior temporal lobe, the parietal-occipital regions, and the rest of the neocortex (3). This distribution of NFTs in AD is classified into 4 stages: B0 (no NFTs), B1 (Braak stages I/II; NFTs predominantly in the entorhinal cortex and closely related areas), B2 (Braak stages III/IV; abundant NFTs in the hippocampus and amygdala, with some extension into the association cortex), and B3 (Braak stages V/VI; NFTs widely distributed throughout the neocortex) (3). Evidence shows that the severity of cognitive impairment in AD parallels the level of neocortical NFT pathology (4), and at least a B2 level of tau pathology with coexisting $\beta$-amyloid and neuritic plaques is necessary to confer a diagnosis of $\mathrm{AD}(3)$.

Recently, the FDA approved ${ }^{18}$ F-flortaucipir, a new PET molecular entity designed to estimate the density and distribution of the aggregated intracellular NFTs (5) in patients with cognitive impairment who are evaluated for AD. For this approval, the FDA relied on the results of 2 clinical studies (NCT02516046 and

Received Jun. 23, 2020; revision accepted Jul. 1, 2020.

For correspondence or reprints contact: Libero Marzella, Center for Drug Evaluation and Research, 10903 New Hampshire Ave., Silver Spring, MD 20993.

Email: libero.marzella@fda.hhs.gov

Published online Aug. 4, 2020.

COPYRIGHT @ 2020 by the Society of Nuclear Medicine and Molecular Imaging. DOI: 10.2967/jnumed.120.252254

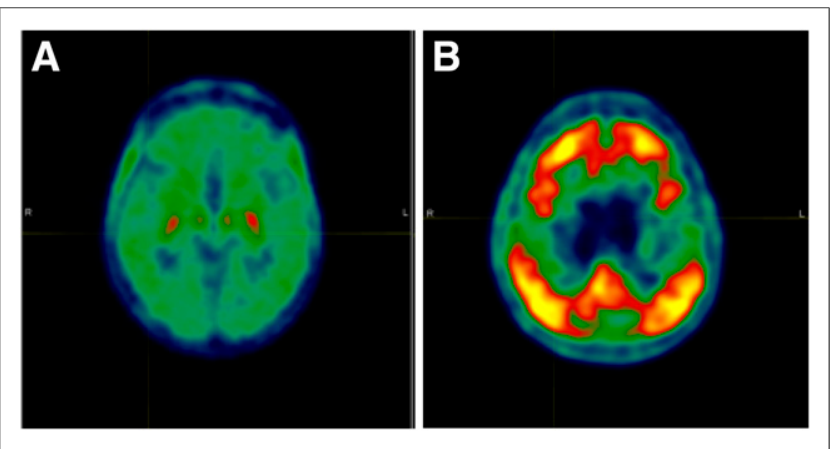

FIGURE 1. Examples of negative and positive ${ }^{18} \mathrm{~F}$-flortaucipir scans. Uptake of ${ }^{18} \mathrm{~F}$-flortaucipir in neocortical gray matter regions only was assessed. (A) Example of negative scan with absence of neocortical activity; off-target binding is noted in choroid plexus and brain stem nuclei. (B) Example of positive scan showing increased neocortical activity in medial prefrontal and cingulate, lateral prefrontal, parietal, occipital, and precuneus regions.

NCT03901092), with ${ }^{18}$ F-flortaucipir scans interpreted by multiple independent readers masked to all clinical information.

The first efficacy study assessed the accuracy of ${ }^{18} \mathrm{~F}$-flortaucipir in estimating the density and distribution of aggregated NFTs in the brains of patients with terminal illness who participated in a postmortem brain donation program. The patients, who had a range of cognitive function, underwent ${ }^{18} \mathrm{~F}$-flortaucipir scanning and were followed until they died. The results of the premortem ${ }^{18} \mathrm{~F}$-flortaucipir scans were compared with the brain tau neuropathology autopsy findings as the truth standard. Diagnostic performance statistics were calculated by defining a positive scan as a B3 level of NFT pathology and a negative scan as a B0, B1, or B2 NFT level.

For the interpretation of the scans, a reader assessed the uptake of ${ }^{18} \mathrm{~F}$-flortaucipir in the neocortical gray matter regions only. A negative scan (e.g., Fig. 1A) typically shows no increased neocortical activity or shows increased neocortical activity isolated to the mesial temporal, anterolateral temporal, or frontal regions. A positive scan (e.g., Fig. 1B) typically shows widespread neocortical activity in the posterolateral temporal, occipital, parietal and precuneus, medial prefrontal and cingulate, and lateral prefrontal regions. The readers showed a high probability for correctly identifying patients with B3 tau pathology (sensitivity across the readers ranged from $92 \%$ [95\% confidence interval, 80\%-97\%] to $100 \%$ [95\% confidence interval, 91\%-100\%]) and an average to high probability for correctly identifying patients without B3 tau pathology 
(specificity across the readers ranged from 52\% [95\% confidence interval, 34\%-70\%] to $92 \%$ [95\% confidence interval, $75 \%-98 \%$ ]).

The second efficacy study assessed interreader agreement. The study included the same patients with terminal illness as in the first study, as well as patients with cognitive impairment being evaluated for AD; the latter represent the indicated population. Agreement across readers for distinguishing positive from negative ${ }^{18} \mathrm{~F}$-flortaucipir scans was high (Fleiss $\kappa$-statistic $=0.87$ [95\% confidence interval, 0.830.91]).

Exploratory analyses of ${ }^{18} \mathrm{~F}$-flortaucipir scans for distinguishing B2-B3 from B0-B1 tau pathology showed numerically lower sensitivity than that for distinguishing B3 from B0-B1-B2 tau pathology. Therefore, patients with clinically meaningful tau pathology (B2) may be missed. These concerns are described in the warnings and precautions section of the prescribing information (5) to caution about the risk of misdiagnosis in patients evaluated for AD. As risk mitigation, an additional evaluation to confirm the absence of AD pathology is recommended in patients with negative ${ }^{18} \mathrm{~F}$-flortaucipir findings. Because $\mathrm{AD}$ is a progressive disease, subsequent testing in patients with worsening cognitive function is likely to demonstrate progression to the B3 level of tau pathology.

In preclinical studies, ${ }^{18} \mathrm{~F}$-flortaucipir was shown to reliably bind to the AD-specific intracellular, phosphorylated, paired helical filamental tau (6). Differences in tau conformation may limit ${ }^{18}$ F-flortaucipir binding in chronic traumatic encephalopathy (7). Therefore, the following limitations-of-use text is included in the prescribing information (5): "TAUVID is not indicated for use in the evaluation of patients for chronic traumatic encephalopathy."

The indication for ${ }^{18} \mathrm{~F}$-flortaucipir can be classified as pathology assessment. This indication is established by demonstrating in a defined clinical setting that a diagnostic radiopharmaceutical has sufficient accuracy in identifying or characterizing the pathology. Further development of tau imaging for the diagnosis and management of patients with cognitive impairment is anticipated. For example, the prognostic usefulness of tau imaging for progression of cognitive and functional impairment due to $\mathrm{AD}$ has not been established and will need to be addressed by further study. The utility of tau imaging for selecting patients and predicting responses to investigational disease-modifying therapies also warrants exploration.
On the background of the approved amyloid PET tracers, FDA approval of ${ }^{18} \mathrm{~F}$-flortaucipir sets the stage for the development of clinical experience with tau pathology assessment vis-à-vis other approaches in the evaluation of patients with cognitive decline.

To summarize, a positive flortaucipir scan indicates the presence of widely distributed tau neuropathology (B3) in the neocortical areas. A negative flortaucipir scan does not rule out the presence of B2 or lower tau pathology or amyloid pathology; an additional evaluation to confirm the absence of AD pathology may be necessary. Subsequent testing in patients with worsening cognitive function may be necessary to detect progression to the B3 level of tau pathology. Important flortaucipir scan limitations are that a positive scan by itself does not establish a diagnosis of AD or other cognitive disorders and that a scan cannot be used in the evaluation of patients for chronic traumatic encephalopathy or other non-AD tauopathies.

\section{DISCLOSURE}

No potential conflict of interest relevant to this article was reported.

\section{REFERENCES}

1. Alzheimer's disease: facts \& figures. BrightFocus Foundation website. https:// www.brightfocus.org/alzheimers/article/alzheimers-disease-facts-figures. Published March 5, 2019. Accessed August 6, 2020.

2. Beach TG, Monsell SE, Phillips LE, Kukull W. Accuracy of the clinical diagnosis of Alzheimer disease at National Institute on Aging Alzheimer Disease Centers, 2005-2010. J Neuropathol Exp Neurol. 2012;71:266-273.

3. Hyman BT, Phelps CH, Beach TG, et al. National Institute on Aging-Alzheimer's Association guidelines for the neuropathologic assessment of Alzheimer's disease. Alzheimers Dement. 2012;8:1-13.

4. Nelson PT, Alafuzoff I, Bigio EH, et al. Correlation of Alzheimer disease neuropathologic changes with cognitive status: a review of the literature. J Neuropathol Exp Neurol. 2012;71:362-381.

5. TAUVID ${ }^{\text {TM }}$ (flortaucipir F 18 injection), for intravenous use [prescribing information]. Food and Drug Administration website. https://www.accessdata.fda.gov/drugsatfda docs/label/2020/212123s000lbl.pdf. Revised May 2020. Accessed August 6, 2020.

6. Lowe VJ, Curran G, Fang P, et al. An autoradiographic evaluation of AV-1451 tau PET in dementia. Acta Neuropathol Commun. 2016;4:58.

7. Marquié M, Aguero C, Amaral AC, et al. $\left[{ }^{18} \mathrm{~F}\right]-\mathrm{AV}-1451$ binding profile in chronic traumatic encephalopathy: a postmortem case series. Acta Neuropathol Commun. 2019;7:164 